\section{Molecular characterization of nephron progenitors and their early epithelial derivative structures in the nephrogenic zone of the canine fetal kidney}

\author{
Rawah Faraj, Angela Irizarry-Alfonzo, \\ Pawan Puri \\ Department of Biomedical Sciences, \\ College of Veterinary Medicine, \\ Tuskegee University, Tuskegee, AL, USA
}

\begin{abstract}
Nephron progenitors (NPs) and nephrogenesis have been extensively studied in mice and humans and have provided insights into the mechanisms of renal development, disease and possibility of NP-based therapies. However, molecular features of NPs and their derivatives in the canine fetal kidney (CFK) remain unknown. This study was focused to characterize the expression of potential markers of canine NPs and their derivatives by immunofluorescence and western blot analysis. Transcription factors (TFs) SIX1 and SIX2, well-characterized human NP markers, were expressed in NPs surrounding the ureteric bud in the CFK. Canine NPs also expressed ITGA8 and NCAM1, surface markers previously used to isolate NPs from the mouse and human fetal kidneys. TF, PAX2 was detected in the ureteric bud, NPs and their derivative structures such as renal vesicle and S-shaped body. This study highlights the similarities in dog, mouse and human renal development and characterizes markers to identify canine NPs and their derivatives. These results will facilitate the isolation of canine NPs and their functional characterization to develop NP-based therapies for canine renal diseases.
\end{abstract}

\section{Introduction}

Understanding of the cellular and molecular basis of renal development can provide insights into the mechanisms of renal disease and facilitate the development of novel regenerative medicine-based therapies. Renal development is a complex process that entails communication among multiple cell types..$^{1-3}$ At the initial stage of renal development, metanephric mesenchyme (MM) containing nephron and stromal progenitors surrounds the ureteric bud. ${ }^{1-3}$ The MM secretes factors that stimulate branching of the ureteric bud, and cells of the ureteric bud induce MM and trans- form MM into cap mesenchyme, a condensed group of NP cells that surround the ureteric bud tips. Both ureteric bud and stromal progenitors contribute to the NP niche that balances self-renewal and differentiation of NPs. ${ }^{4,5}$ Cells of the ureteric bud give rise to collecting ducts whereas, stromal progenitors generate vascular and interstitial cells. NPs give rise to all cell types of the nephron. ${ }^{3}$

NPs, a multipotent cell population possessing full nephron-forming potential, can be identified by SIX2 and/or Cited1 expression. ${ }^{6,7}$ SIX2 plays an indispensable role in renal development and controls NP cell fate by regulating self-renewal $v s$ differentiation decisions; whereas, deletion of Cited 1 does not impact NP compartment and renal development. ${ }^{6-8}$ During nephrogenesis, mesenchymal NPs undergo mesenchymal to epithelial transition (MET) and generate the renal vesicle that develops first into a comma-shaped body, and then, into a Sshaped body. ${ }^{1-3}$ S-shaped body consists of proximal, intermediate, distal and connecting segments made up of distinct precursor cell types that give rise to different nephron segments such as glomerulus, proximal convoluted tubule, Loop of Henle, distal convoluted tubule and connecting segment. ${ }^{2,9}$ In-depth analyses using genetic, molecular and cellular approaches in mouse models, have defined the molecular signatures of various specialized cell types in different stages of nephron development and the molecular signals that regulate nephrogenesis. ${ }^{10,11}$ Furthermore, multiple groups have explicated methodology to isolate and expand mouse NP population in synthetic niches as well as elucidated strategies to coax these in vitro maintained NPs to differentiate into glomerular and tubular cell types and assemble into kidney organoids. ${ }^{4,12,13}$

With the fundamental knowledge obtained from mouse models, NPs from human developing kidneys have been isolated and maintained in culture. ${ }^{13,14}$ Furthermore, human NPs have been generated from induced pluripotent stem cells and developed into differentiated kidney cells and kidney organoids..$^{15-17}$ Recent studies reported detailed molecular characterization of human NPs, their nephrogenic niche and nephron patterning in the human embryonic and fetal kidneys in various stages of development. ${ }^{18-24}$ These studies observed strong conservation in molecular markers of NPs and their derivatives as well as in pathways regulating nephrogenesis between developing mouse and human kidneys; although, a few distinct features for each species were determined as well. ${ }^{19-23}$ Together, these experimental studies on mouse and human NPs and their develop-
Correspondence: Pawan Puri, Department of Biomedical Sciences, Tuskegee University College of Veterinary Medicine, A310 Patterson Hall, Tuskegee, AL 36088, USA. Tel. +1.334.724-4486 - Fax: +1.334.7278177. E-mail: ppuri@tuskegee.edu

Key words: Nephron progenitor; renal development; nephrogenesis.

Authors' contributions: RF, AIA, Western blots analyses; PP, study design, co-immunofluorescence performing, contribution to the immunoblot experiments, manuscript drafting. All the authors read and approved the final version of the manuscript.

Conflict of interests: The authors declare that they have no competing interests.

Acknowledgements: We thank Dr. Rachel Tears from Alabama Animal Alliance Spay Neuter Clinic for providing gravid uteri. We also thank Dr. Gopal Reddy (Professor, Tuskegee University College of Veterinary Medicine - TUCVM) for sharing research supplies. We also thank RCMI Microscope Facility, and Anatomic Pathology Facility at TUCVM. We thank Dr. William H Walker (Associate Professor, University of Pittsburgh) for his help in editing the manuscript.

Funding: This research was supported by funds provided by the Tuskegee University College of Veterinary Medicine (PP). Research in principal investigator's laboratory is supported by funding from the 1SC2GM130475-01(PP) grant awarded by the National Institute of General Medical Sciences. AIA was supported by NIH/SRE 5T35OD010432.

Received for publication: 3 July 2019.

Accepted for publication: 10 September 2019.

This work is licensed under a Creative Commons Attribution-NonCommercial 4.0 International License (CC BY-NC 4.0).

(C) Copyright: the Author(s), 2019

Licensee PAGEPress, Italy

European Journal of Histochemistry 2019; 63:3040 doi:10.4081/ejh.2019.3049

ment into nephrons have strong implications in the development and translation of regenerative medicine-based approaches to treat diseases requiring nephron repair and regeneration such as chronic kidney disease (CKD), increase the efficiency of de novo nephrogenesis and model various other renal diseases. ${ }^{25,26}$

In dogs, CKD has reported prevalence of up to $25 \%$ and is one of the major causes of morbidity and mortality. ${ }^{27-29}$ Nephron loss is one of the main underlying causes of progressive decline in renal function in 
CKD and ultimately leads to renal failure. ${ }^{30}$ Transplantation of NPs isolated from the embryonic kidney have been shown to repair and enhance renal function in rodent models of renal injury and such NP-based therapeutic strategies have potential to address nephron loss in CKD in companion animals. ${ }^{13,31,32}$ However, markers to identify and isolated canine NPs and mechanisms regulating their maintenance and differentiation into nephrons are completely unknown. This knowledge can not only help in developing novel strategies to treat CKD in dogs but also help understand the molecular basis of congenital canine diseases such renal and cystic dysplasia. ${ }^{33-35}$ Furthermore, dogs with CKD can also serve as a large animal model for preclinical studies to test the therapeutic potential of these NP-based regenerative approaches once the fundamental knowledge about molecular mechanisms regulating canine NPs and nephrogenesis are elucidated. In the current study, we performed molecular characterization of canine NPs and their derivates in the canine fetal kidney (CFK) and found strong conservation in their expression pattern between canine, mouse and human developing kidney. This study provides a framework to identify and isolate canine NPs and will facilitate future studies for functional characterization of canine NPs. cence studies, rehydrated sections were subjected to boiling for antigen retrieval in sodium citrate buffer $(10 \mathrm{mM}$ sodium citrate, $0.1 \%$ Tween-20, $\mathrm{pH} 6.0$ ) at $95^{\circ} \mathrm{C}$ for 30 min and then kept at room temperature for $30 \mathrm{~min}$. The sections following antigenretrieval were blocked in donkey serum for at least $1 \mathrm{~h}$ at room temperature and incubated overnight with following primary antibodies: E-cadherin (ECAD) (1:1000, Cat \#610181; BD Biosciences, Franklin Lakes, NJ, USA), NCAM1 (1:1000, 3576; Cell Signaling Technology, Danvers, MA, USA), N-cadherin (NCAD) (1:1000, 13116; Cell Signaling), PAX2 (1:1000, 71-6000; Thermo Fisher, Rockford, IL, USA), SIX1 (1:1000, 16960; Cell Signaling), SIX2 (1:1000, 11562-1-AP; Protein Tech, Rosemont, IL, USA) and ITGA8 (1:500, AF4076; R\&D Systems, Minneapolis, MN, USA), Alexa Fluor 594 and Alexa Fluor 488 secondary antibodies were used and nuclei were stained with 4',6-diamidino-2-phenylindole. Staining was detected using Olympus fluorescence BX53F microscope (Leica, Wetzlar, Germany).

\section{Preparation of whole cell extract from CFK and immunoblotting}

To prepare whole cell lysates, kidneys isolated from dog fetuses were homogenized in RIPA lysis buffer supplemented with a protease inhibitor cocktail and rocked for $30 \mathrm{~min}$ at $4^{\circ} \mathrm{C}$ to extract proteins. To remove cell debris, solution was pelleted by centrifugation at $14,000 \mathrm{x}$ g for $20 \mathrm{~min}$ and supernatant was used for immunoblotting. Cell lysates were fractionated by SDSPAGE, transferred to nitrocellulose membranes, and incubated with following primary antibodies at following dilutions: ECAD (1:1000), NCAM1 (1:2000), NCAD (1:1000), PAX2 (1:1000), SIX1 (1:1000), SIX2 (1:1000), and ITGA8 (1:1000). Following washing, membranes were incubated with secondary antibody conjugated with horseradish peroxidase. The proteinantibody complex was visualized with SignalFire Elit Chemiluminescent HRP substrate (Cell Signaling).

\section{Results}

\section{H\&E staining of the CFK}

First, CFK sections were stained with $\mathrm{H} \& \mathrm{E}$ to detect and assess NPs and other developing structures in the nephrogenic zone. Low magnification analysis revealed the nephrogenic zone in the outer renal cortex constituted predominantly by heavily hematoxylin-stained cells (Figure 1A).

\section{Materials and Methods}

\section{Fetal kidney tissue isolation}

CFKs were collected from 11 fetuses isolated from gravid uteri isolated from three pregnant mixed breed dogs of more than 2 years of age presented from elective spay in Alabama Animal Alliance Spay Neuter Clinic, Montgomery. All the females were estimated to be in third trimester of pregnancy based upon the development of reproductive organs and claws of the fetuses. ${ }^{36,37}$ The collection and use of the tissuematerial for the current study was reviewed and approved by Tuskegee University Institutional Animal Care and Use Committee (TUIACUC) and performed according to institutional guidelines.

\section{Hematoxylin \& Eosin staining and immunofluorescence studies}

Histological and immunofluorescence protocols used in the current study have been described previously. ${ }^{38-42}$ Briefly, isolated CFKs were fixed in $4 \%$ paraformaldehyde and embedded in paraffin; 5 - $\mu$ m thick sections were prepared and were deparaffinized, rehydrated and subjected to Hematoxylin \& Eosin (H\&E) staining with the routine protocol. For immunofluores-

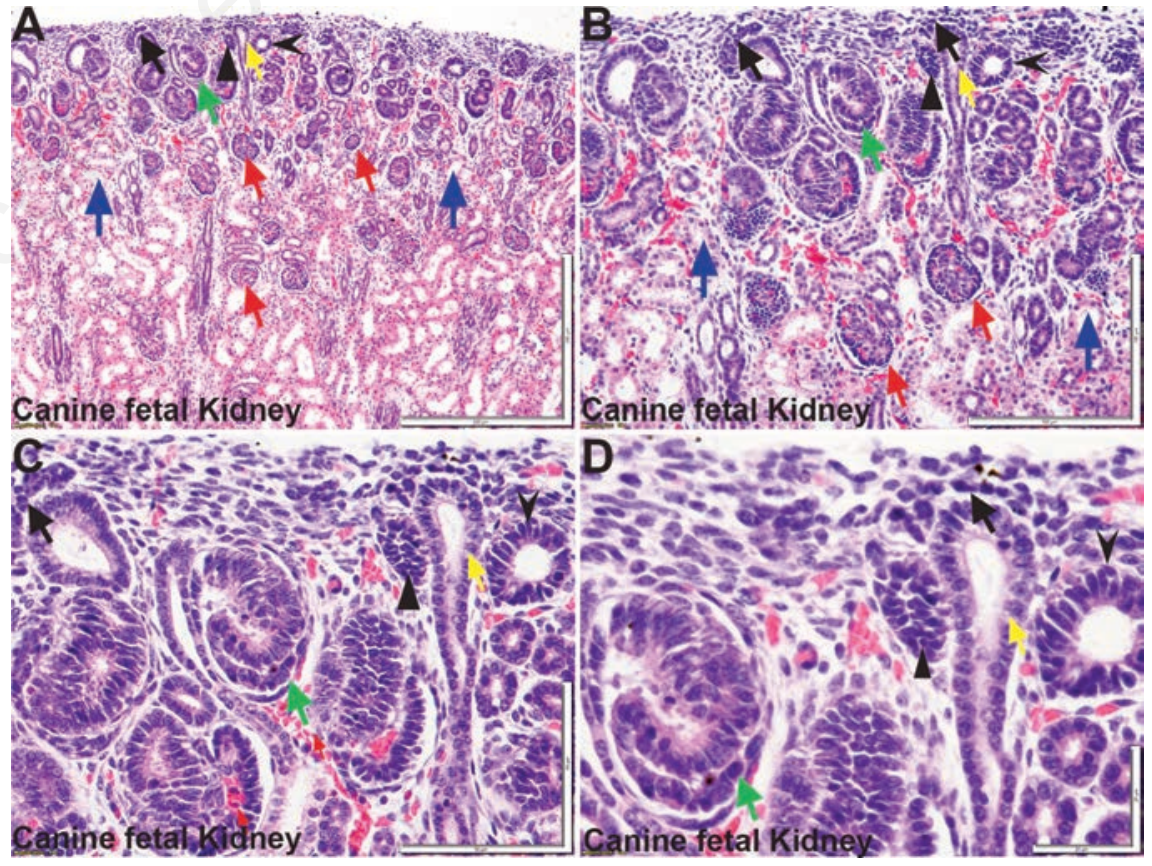

Figure 1. Histological analyses of the CFK. A-D) Representative images of H\&E stained sections of dog fetal kidney at low $(10 \mathrm{X})$ and high $(20 \mathrm{X}, 40 \mathrm{X}$ and $60 \mathrm{X})$ magnification showing developing structures in the nephrogenic zone. A-D) Black arrows indicate the NP population surrounding the ureteric bud (yellow arrow); arrowheads indicate pretubular aggregates; concave arrowheads indicate renal vesicle; green colored arrows indicate $S$-shaped body; red arrows indicate glomeruli whereas developing tubules are indicated by blue arrows (A, B). Scale bars: A) $200 \mu \mathrm{m}$; B) $100 \mu \mathrm{m}$; C) $50 \mu \mathrm{m}$; D) $20 \mu \mathrm{m}$. 
High magnification analysis revealed condensed cap mesenchymal cells, known to have nephron-forming potential, surrounding the ureteric bud tips (Figure 1 B-D). A subpopulation of NPs becomes committed and give rise to pretubular aggregates (PTA) that further differentiate into renal vesicles. Both PTA and renal vesicle could be seen on the sides and/or underneath the ureteric bud tips in the H\&E stained sections (Figure 1 B-D). Analysis also revealed comma and S-shaped bodies, developing underneath the ureteric bud tips (Figure 1 B-D). Below the nephrogenic zone, developing renal corpuscles and tubular segments in different stages of maturation could be visualized (Figure 1 B-D). These results reveal similar progression and structural features of nephrogenesis in CFK as previously reported in the mouse and human fetal kidney. ${ }^{3,22}$

\section{SIX2/ECAD co-immunofluorescence analysis}

SIX2 is a well-established marker of mesenchymal NPs that is highly conserved from mouse to human. ${ }^{6,21,43}$ ECAD is expressed by the ureteric bud and NP-derivatives that are in the process of acquiring epithelial identity. To determine whether SIX2 and ECAD are expressed in the CFK, we performed western blot analysis. Both SIX2 and ECAD proteins were detected in the CFK by immunoblotting (Figure $2 \mathrm{~A}, \mathrm{~B}$ ). To determine whether SIX2 is expressed in mesenchymal nephron progenitors and how its expression changes in NP-derivates in the embryonic dog kidney, we performed co-immunofluorescence studies using antibody against SIX2 along with ECAD antiserum. For a negative control, staining with isotype control antibodies was performed that did not show any signal (Figure 2C). In sections, co-stained with SIX2 and E cadherin, strong SIX2 signal was specifically detected in the few layers of condensed mesenchymal cells surrounding the ureteric bud; and as expected, the cells with strong SIX2 signal did not show ECAD expression (Figure 2 D-F). SIX2 signal was specifically localized to the nucleus of NPs. In both pretubular aggregates and renal vesicles in the embryonic dog kidney, SIX2 expression decreased and ECAD signal could be detected indicating the advent of epithelization (Figure 2 D-F). SIX2 signal was undetectable beyond the renal vesicle stage. Ureteric bud cells that strongly expressed
ECAD did not express SIX2. These results show that SIX2 marks mesenchymal canine NPs and has similar protein distribution in CFK as previously reported in mouse and human fetal kidney. ${ }^{6,43,44}$

\section{SIX2/NCAM1 co-immunofluores- cence analysis}

NCAM1 is known to be expressed in NPs and their early epithelial derivatives; furthermore, its membrane localization makes it a suitable NP marker to sort cells with nephron-forming potential..$^{32,41,45}$ First, we determined by immunoblot analysis that NCAM1 is expressed in the CFK (Figure 3A). To determine whether SIX2-positive NPs also express NCAM1 and study the expression of NCAM1 in the early epithelial structures developing in the nephrogenic zone in the CFK, co-immunofluorescence analysis with SIX2 and NCAM1 antisera was performed. Co-staining analysis revealed that a subpopulation of highexpressing SIX2-positive cells expresses NCAM1 at their membrane (Figure 3 B-D). As NPs differentiate into a renal vesicle, SIX2 expression was dramatically reduced whereas, a strong membrane NCAM1 signal was detected in these cells. NCAM1
A

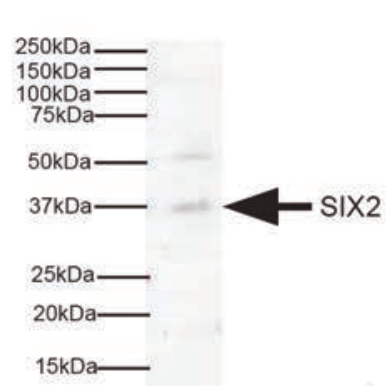

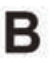

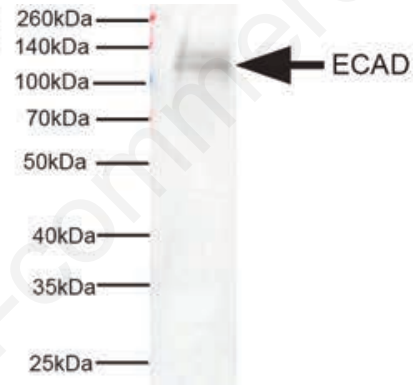

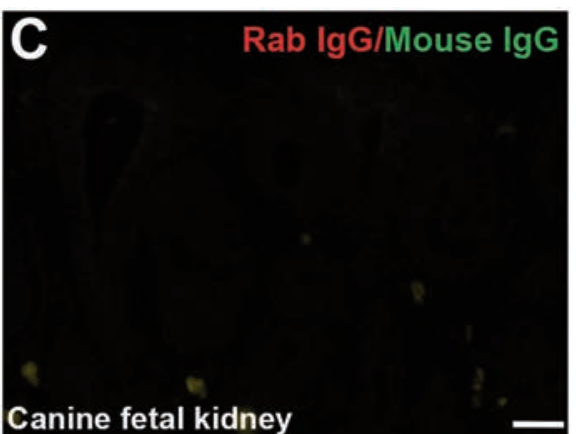
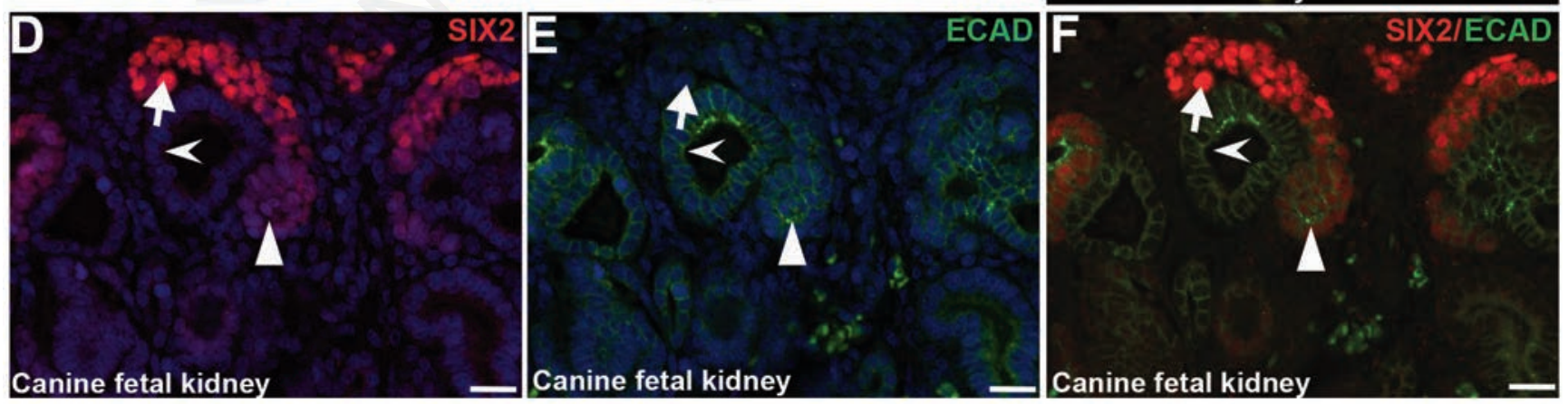

Figure 2. Expression of SIX2 and ECAD in the CFK. A,B) Whole kidney extracts were prepared from CFK and subjected to Immunoblot analysis with antiserum against SIX2 and ECAD antisera. C-F) Representative co-immunofluorescence images of CFK section stained with isotype control antibodies (C) or SIX2 (red) and ECAD (green) antisera (D-F); nuclei were stained with DAPI. Strong SIX2 signal (red) (arrow) (D) was observed in the NP population that did not express ECAD (green) (arrow) (E); shown also on the merged image (arrow) (F); a weaker SIX2 signal was observed in pretubular aggregates (arrowhead) (D) that showed weak ECAD signal (arrowhead) (E); also shown on the merged image (arrowhead) (F); ECAD expression (concave arrowhead) (E) was observed in the ureteric bud that did not express SIX2 (concave arrowhead) (D); also shown on merged image (concave arrowhead) (F). Scale bars: $20 \mu \mathrm{m}$. 
expression was also observed in proximal, intermediate and distal segments of the Sshaped bodies (Figure 3 B-D). In S-shaped bodies, NCAM1 signal was undetectable in podocyte precursors. Cell-surface localization of NCAM1 in SIX2-positive NP indicates that NCAM1 could be exploited to sort canine NPs. However, gentle dissociation of NPs present on the cortical surface of the kidney will be required to avoid contamination from the relatively deeper layers of NCAM1-positive early epithelial structures during sorting.

\section{SIX1/ ECAD and SIX1/NCAM1} co-immunofluorescence analysis

SIX1 is expressed in the MM of both mouse and human kidney. Notably, SIX1 expression continues in the cap mesenchyme (NPs) of the human kidney however, its expression becomes undetectable in the cap mesenchyme (NPs) of the mouse kidney. ${ }^{46}$ Western blot analysis with SIX1 antiserum showed that SIX1 protein is expressed in the CFK (Figure 4A). To determine the expression of SIX1 in NPs and their early epithelial derivate structures in the nephrogenic zone of the dog fetal kidney co-immunofluorescence analyses of
SIX1 antibody with ECAD or NCAM1 antisera were performed. Strong nuclear SIX1 signal was detected in cap mesenchymal cells surrounding the ECAD-positive ureteric bud cells (Figure 4 B-D). SIX1 expression continued. in the pretubular aggregate and renal vesicle, structures identified by the expression of NCAM1 (Figure 4 E-G). In the distal segment of the Sshaped body, a weak SIX1 signal could be detected. These results indicate that the SIX1 distribution in CFK is similar to its reported distribution in the human fetal kidney but, is strikingly different from the mouse fetal kidney that does not express SIX1 in NPs. ${ }^{46}$

\section{PAX2/ECAD and PAX2/NCAM1 co- immunofluorescence analyses}

PAX2 expression has been reported in NPs and their derivatives as well as in the ureteric bud; and PAX2 expression is essential for renal development in both lineages. ${ }^{47-49}$ To determine whether PAX2 protein is expressed in the CFK, we performed immunoblotting analysis on whole cell extracts prepared from the dog fetal kidney. PAX2 protein expression could be detected by western blot analysis (Figure 5A). PAX2 expression was detected in the ECAD-positive ureteric bud and cap mesenchymal cells surrounding the ureteric bud in the CFK (Figure 5 B-D). Strong PAX2 expression was also detected in NP-derivatives pretubular aggregates, renal vesicles and $\mathrm{S}$ shaped bodies, structures identified by the expression of NCAM1 (Figure 5 E-G). PAX2 was localized to the nucleus in NPs and their derivatives and in the cells of the ureteric bud. PAX2 expression became undetectable in proximal tubules and distal tubules (not shown) however, PAX2 expression was detectable in differentiated collecting ducts in the medulla (not shown). Together, these results indicate that expression and localization of PAX2 in CFK is similar to human and mouse kidney. ${ }^{47}$

ITGA8/ECAD and ITGA8/NCAM1 co-immunofluorescence analyses

ITGA8 is a surface marker expressed in human and mouse kidney and used to purify cap mesenchymal cells with nephron-forming potential. ${ }^{46,50,51}$ Immunoblot analysis with ITGA8 antiserum showed ITGA8 protein expression in the CFK (Figure 6A). Coimmunofluorescence analysis with ITGA8 antibody with Ecadherin or NCAM1 anti-
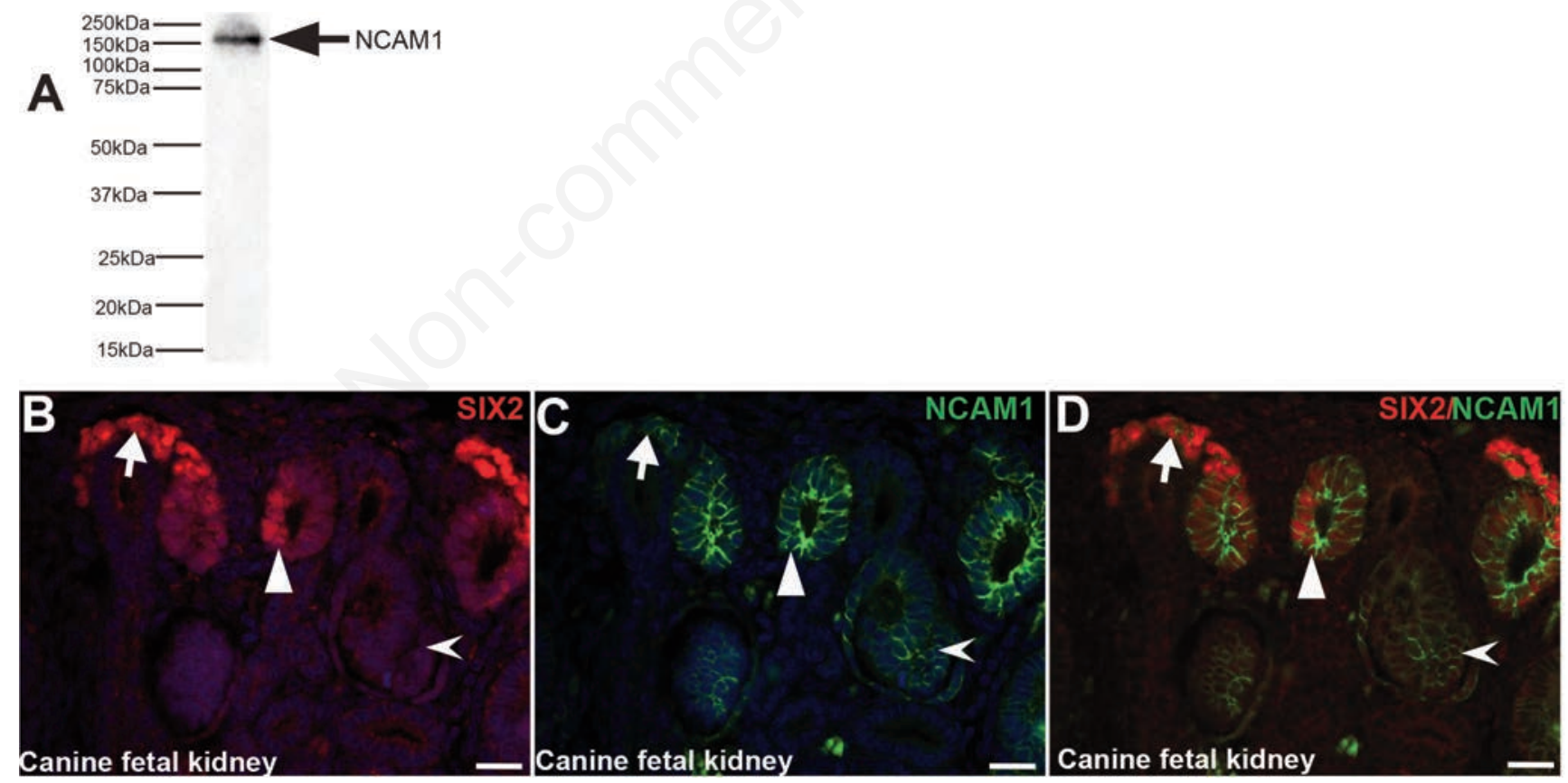

Figure 3. Expression of SIX2 and NCAM1 in the CFK. A) Whole kidney extracts were prepared from CFK and subjected to Immunoblot analysis with antiserum against NCAM1. B-D) Representative co-immunofluorescence images of CFK sections with SIX2 (red) and NCAM1 (green) antisera; nuclei were stained with DAPI; a subpopulation of SIX2-positive (red) (arrow) (B) expressed NCAM1 (green) (arrow) (C); also shown on the merged image (arrow) (D); a weaker SIX2 (arrowhead) (B) signal was observed in the renal vesicle cells that expressed strong NCAM1 signal (arrowhead) (C); also shown on the merged image (arrowhead) (D); NCAM1 expression (concave arrowhead) (C) was observed in NP-epithelial derivate structure, S-shaped body that did not express SIX2 (concave arrowhead) (B); also shown on the merged image (concave arrowhead) (D). Scale bars: $20 \mu \mathrm{m}$. 
sera was performed to determine its expression and distribution in the nephrogenic zone of the CFK. As a negative control, staining with isotype control (mouse and goat) antibodies was performed that did not show any signal (Figure 6B). ITGA8 signal was absent in the ECAD-positive ureteric bud (Figure $6 \mathrm{C}-\mathrm{E}$ ). A strong ITGA8 signal was found at membrane of NPs cells that expressed NCAM1 (Figure 6 F-H). ITGA8 was also expressed in pre-tubular aggregates and renal vesicles (identified by ECAD and NCAM1 expression) albeit, at lower level (Figure $6 \mathrm{C}$-H). Very faint ITGA8 expression could also be detected in the distal segment of S-shaped body. ITGA8 expression was found in the mesangial cells of the developing and developed renal corpuscles (not shown). Given that ITGA8 is highly expressed and localized at the membrane in canine NPs and, its expression is relatively faint/weak in the early epithelial structures indicates that ITGA 8 is very likely a suitable marker for the isolation of NPs from CFK.

\section{NCAD/ECAD and NCAD/NCAM1 co-immunofluorescence analyses}

NCAD, a mesenchymal stem cell marker is known to be expressed in mouse NPs however, its functional significance in the renal development remains unclear. ${ }^{52,53}$ Western blot analysis with NCAD antiserum showed that NCAD protein is expressed in the CFK (Figure 7A). Coimmunofluorescence analysis of $\mathrm{CFK}$ with NCAD antibody and ECAD or NCAM1 antisera was performed. NCAD signal was

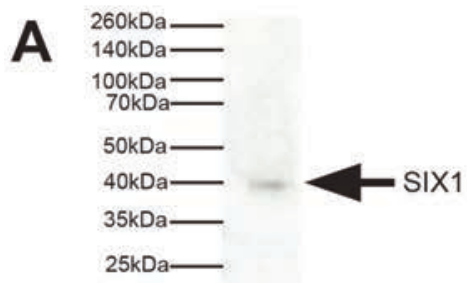

$15 \mathrm{kDa}$
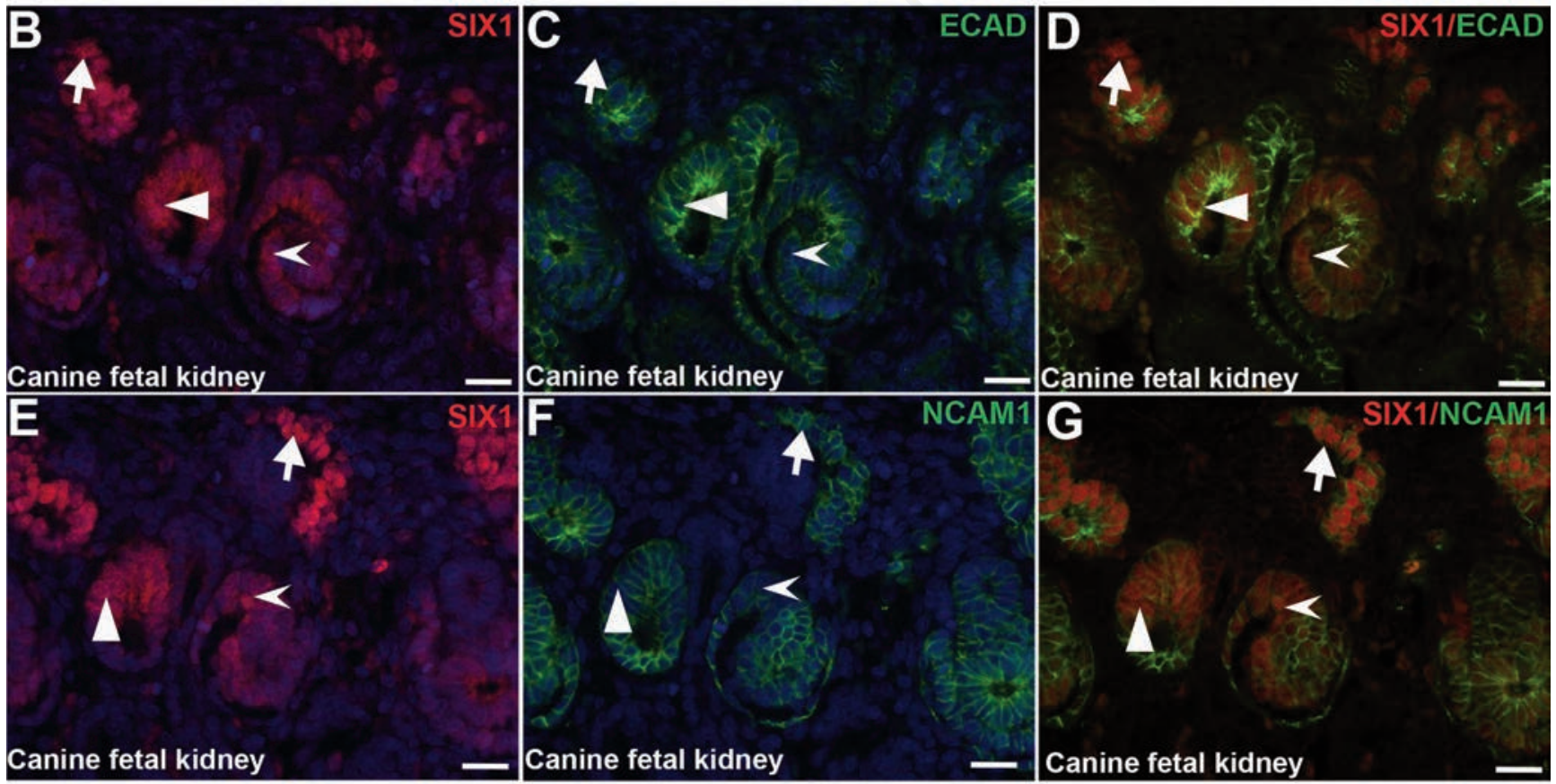

Figure 4. Expression of SIX1 in the CFK. A) Whole kidney extracts were prepared from CFK and subjected to Immunoblot analysis with antiserum against SIX1 antiserum. B-D) Representative co-immunofluorescence images of CFK sections with SIX1 (red) and ECAD (green) antisera; nuclei were stained with DAPI; a strong SIX1 signal (red) (arrow) (B) was observed in the NP population that did not express ECAD (green) (arrow) (C); shown also on the merged image (arrow) (D); SIX1 expression was observed in the renal vesicle (arrowhead) (B) cells that showed strong ECAD signal (arrowhead) (C); also shown on the merged image (arrowhead) (D); a weak and patchy SIX1 expression (concave arrowhead) (B) was observed in the S-shaped body that also expressed ECAD (concave arrowhead) (C); also shown on merged image (concave arrowhead) (D). E-G) Representative co-immunofluorescence images of CFK sections with SIX1 (red) and NCAM1 (green) antisera. SIX1-positive cells (red) (arrow) (E) expressed NCAM1 (green) (arrow) (F); also shown on the merged image (arrow) (G); SIX1 expression was observed in the renal vesicle cells (concave arrowhead) (E) that expressed strong NCAM1 signal (arrowhead) (F); shown also on the merged image (arrowhead) (G); a few SIX1-positive cells (concave arrowhead) (E) were observed in the S-shaped body identified by NCAM1 expression (concave arrowhead) (F); also shown on the merged image (concave arrowhead) (G). Scale bars: $20 \mu \mathrm{m}$. 
absent in the ECAD-positive ureteric bud (Figure 7 B-D). Strong NCAD signal was found at membrane of cap mesenchymal cells and pretubular aggregates identified by NCAM1 (Figure 7 E-G). A weak NCAD signal could be detected in the NCAM1positive S-shaped body.

\section{Discussion}

In this study, molecular characterization of NPs and their early epithelial derivatives in the nephrogenic zone of the CFK with histological and immunofluorescence-based studies were performed. Specifically, this study describes the expression pattern of multiple transcription factors in the fetal kidney of dogs that have found to be important for the maintenance of NPs in mouse and human. This study also describes the expression pattern of various surface markers in canine NPs that have been used to isolate and enrich murine and human NPs.

SIX2 is a well-characterized and specific NP-marker and has a similar pattern of protein distribution and localization in the developing mouse and human kidney. ${ }^{6,21,44,46}$ The expression pattern of SIX2 in canine NPs and their early derivates was found to be highly similar to its expression reported in mouse and human developing kidney. ${ }^{54}$ SIX2 expression level has been used as a benchmark to gauge the purity and maintenance of NP-identity in various in vitro culture conditions. ${ }^{4,7,13,14}$ Notably, SIX2 RNAprobe has been successfully used to sort NPs from the human kidney. ${ }^{14}$ Given the conserved expression pattern of SIX2 in
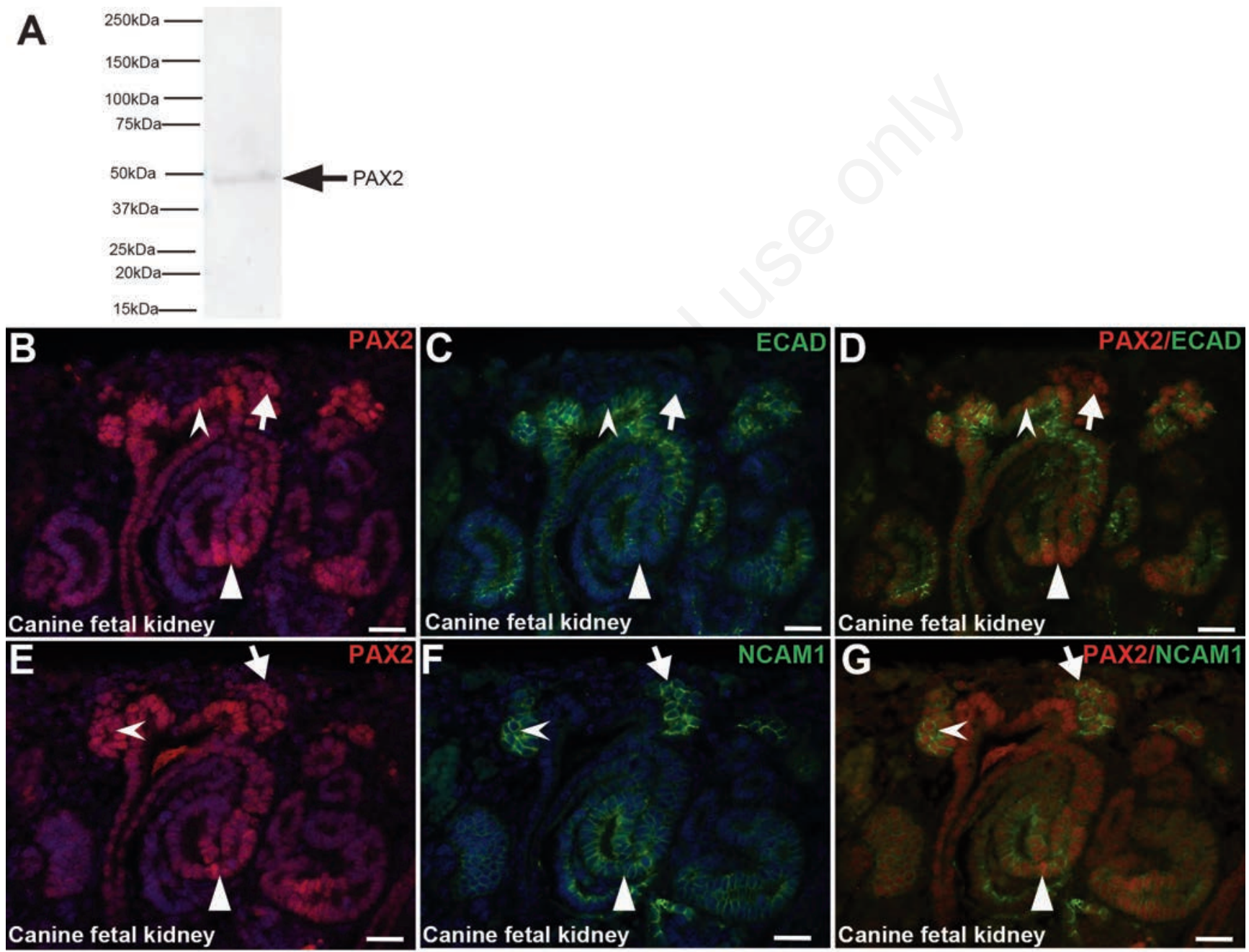

Figure 5. Expression of PAX2 in the CFK. A) Whole kidney extracts were prepared from CFK and subjected to Immunoblot analysis with antiserum against PAX2 antiserum. B-D) Representative co-immunofluorescence images of CFK sections with PAX2 (red) and ECAD (green) antisera; nuclei were stained with DAPI; a strong PAX2 signal (red) (arrow) (B) was observed in the NP population that did not express ECAD (green) (arrow) (C); shown also on the merged image (arrow) (D); PAX2 expression was observed in the S-shaped body (arrowhead) (B) cells that also expressed ECAD (arrowhead) (C); also shown on the merged image (arrowhead) (D); PAX2 expression (concave arrowhead) (B) was also observed in the ECAD-positive ureteric bud (concave arrowhead) (C); also shown on the merged image (concave arrowhead) (D). E-G) Representative co-immunofluorescence images of CFK sections with PAX2 (red) and NCAM1 (green) antisera; a subpopulation of PAX2-positive (red) (arrow) (E) expressed NCAM1 (green) (arrow) (F); also shown on the merged image (arrow) (G); a strong PAX2 (concave arrowhead) (E) signal was observed in the renal vesicle cells that expressed strong NCAM1 signal (concave arrowhead) (F); also shown on the merged image (concave arrowhead) (G); PAX2 expression (arrowhead) (E) was observed in NP-epithelial derivate structure, the S-shaped body identified by NCAM1 expression (arrowhead) (F); also shown on the merged image (arrowhead) (G). Scale bars: $20 \mu \mathrm{m}$. 
canine, mouse and human fetal kidney, the results of this study indicate that canine SIX2-specific RNA-probes could be used to isolate canine NPs and its NP-specific marker status could be exploited to gauge maintenance of NP identity in while testing various in vitro culture conditions for NPs.

In contrast to SIX2, the expression pattern of SIX1, a SIX2-related family member, in human and mice developing kidney is interestingly different. ${ }^{46,54}$ SIX1 expression in mice is restricted to the MM in the early stages of renal development and becomes undetectable in NPs. ${ }^{54}$ In the human kidney, SIX1 is expressed in the $\mathrm{MM}$ and its expression is maintained in the NP population. ${ }^{46,54}$ In the human developing kidney, combined expression of SIX1 and SIX2 has been proposed to support NP selfrenewal and maintain nephrogenesis for a relatively longer gestation length in humans. ${ }^{46}$ The combined expression of SIX1 and SIX2 has also been proposed to contribute to the generation of higher nephron number (average 1 million) in humans as compared to mice (average
20,000)..${ }^{54}$ Similar to human, in canine kidney, both SIX1 and SIX2 expression were expressed at high levels in NPs of fetal kidney and may contribute to maintenance of NPs and generation of higher number of nephrons. The average number of nephrons in the adult canine kidney has been estimated to approximately 475,000 that develop in approximately 50-60 days. ${ }^{55}$ It would be interesting to determine the role of SIX1 and SIX2 in the self-renewal of NPs in an in vitro canine NP culture model.

PAX2 expression pattern in the CFK
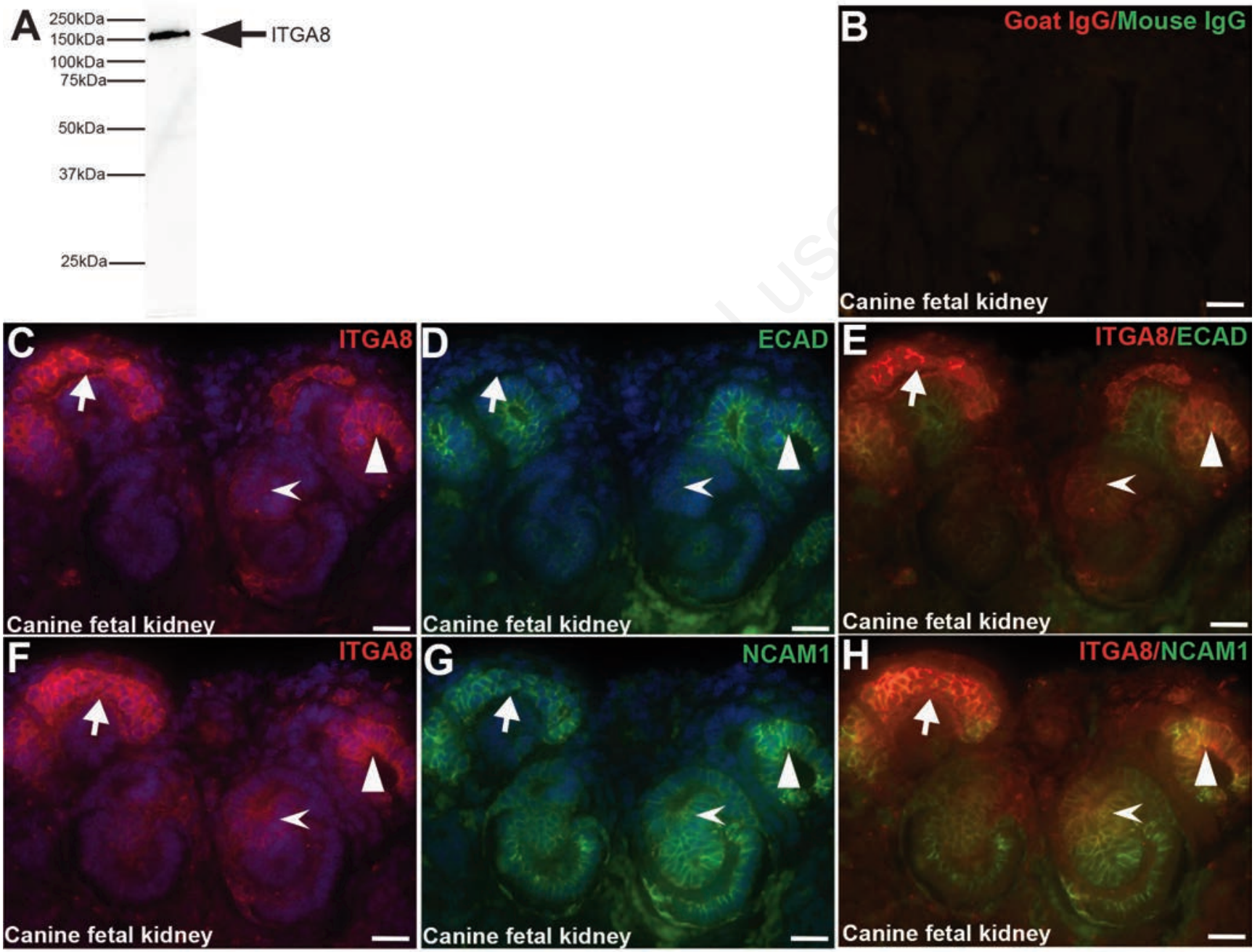

Figure 6. Expression of ITGA8 in the CFK. A) Whole kidney extracts were prepared from CFK and subjected to Immunoblot analysis with antiserum against ITGA8 antiserum. B-E) Representative co-immunofluorescence images of CFK sections with isotype control antibodies (B) or ITGA8 (red) and ECAD (green) antisera; nuclei were stained with DAPI; a strong ITGA8 signal (red) (arrow) (C) was observed on surface of NP population that did not express ECAD (green) (arrow) (D); also shown on the merged image (arrow) (E); ITGA8 expression (arrowhead) (C) in the ECAD-positive renal vesicle (arrowhead) (D); also shown on the merged image (arrowhead) (E); a weaker ITGA8 signal (concave arrowhead) (C) was detected in the distal segment of the ECAD-positive S-shaped body (concave arrowhead) (D); also shown on the merged image (concave arrowhead) (E). F-H) Representative co-immunofluorescence images of CFK sections with ITGA8 (red) and NCAM1 (green) antisera; a subpopulation of ITGA8-positive (red) (arrow) (F) expressed strong NCAM1 signal (green) (arrow) (G); also shown on the merged image (arrow) (H); ITGA8 signal (arrowhead) (F) in the NCAM1-positive renal vesicle (arrowhead) (G); also shown on the merged image (arrowhead) (H). ITGA8 expression (concave arrowhead) (F) in the distal segment of the S-shaped body identified by NCAM1 expression (concave arrowhead) (G); also shown on the merged image (arrowhead) (H). Scale bars: $20 \mu \mathrm{m}$. 
has strong similarities to its expression pattern in the mouse and human developing kidney. ${ }^{47}$ PAX2 plays indispensable roles in various stages of renal development in multiple cell types. ${ }^{56}$ Global deletion of Pax2 results in renal agenesis due to degeneration of the nephric ducts. ${ }^{56-58}$ PAX2 regulates the outgrowth of the ureteric bud by regulating glial derived nerve growth factor (GDNF) and c-Ret expression. ${ }^{49,56,59}$ PAX2 also regulates branching morphogenesis of the ureteric bud; mice expressing one mutant allele of Pax $2^{1 \mathrm{Neu}}$, show reduced branching of the ureteric bud which contributes to renal hypoplasia. ${ }^{56,60} \mathrm{PAX} 2$ regulates differentiation of NPs, by regulating mesenchy- mal to epithelial transition. ${ }^{49,56,61}$ Recently, it has been shown that PAX2 in NPs is essential to maintain their identity and PAX2-deficent NPs transdifferentiate into interstitiallike cells. ${ }^{47}$ In CFK, PAX2 is localized in NPs and their early epithelial derivates as well as in the ureteric bud. The role of PAX2 in the renal development of dogs remains to be determined.

Notably, PAX2 mutations cause renal coloboma syndrome in humans. ${ }^{62,63}$ SIX2 mutations cause renal hypodysplasia and SIX1 mutations are known to cause brachio-oto-renal syndrome in humans. ${ }^{43,54}$ Knockout mouse models have also identified indispensable roles of these three genes in the renal development. ${ }^{6,47,64}$ In dog, renal dysplasia cases are known to occur frequently; however, its molecular basis is not well understood. ${ }^{28,33,35}$ This study reports the expression pattern of transcription factors SIX2, SIX1 and PAX2 in the CFK and highlights the conserved and distinct features in relation to the human and mouse kidney. These genes could be carefully analyzed in dogs while screening cases of renal maldevelopment to determine candidate genes for renal dysplasia.

Development and standardization of strategies for the isolation and expansion of canine NPs are essential to develop NPbased therapeutic approaches for canine
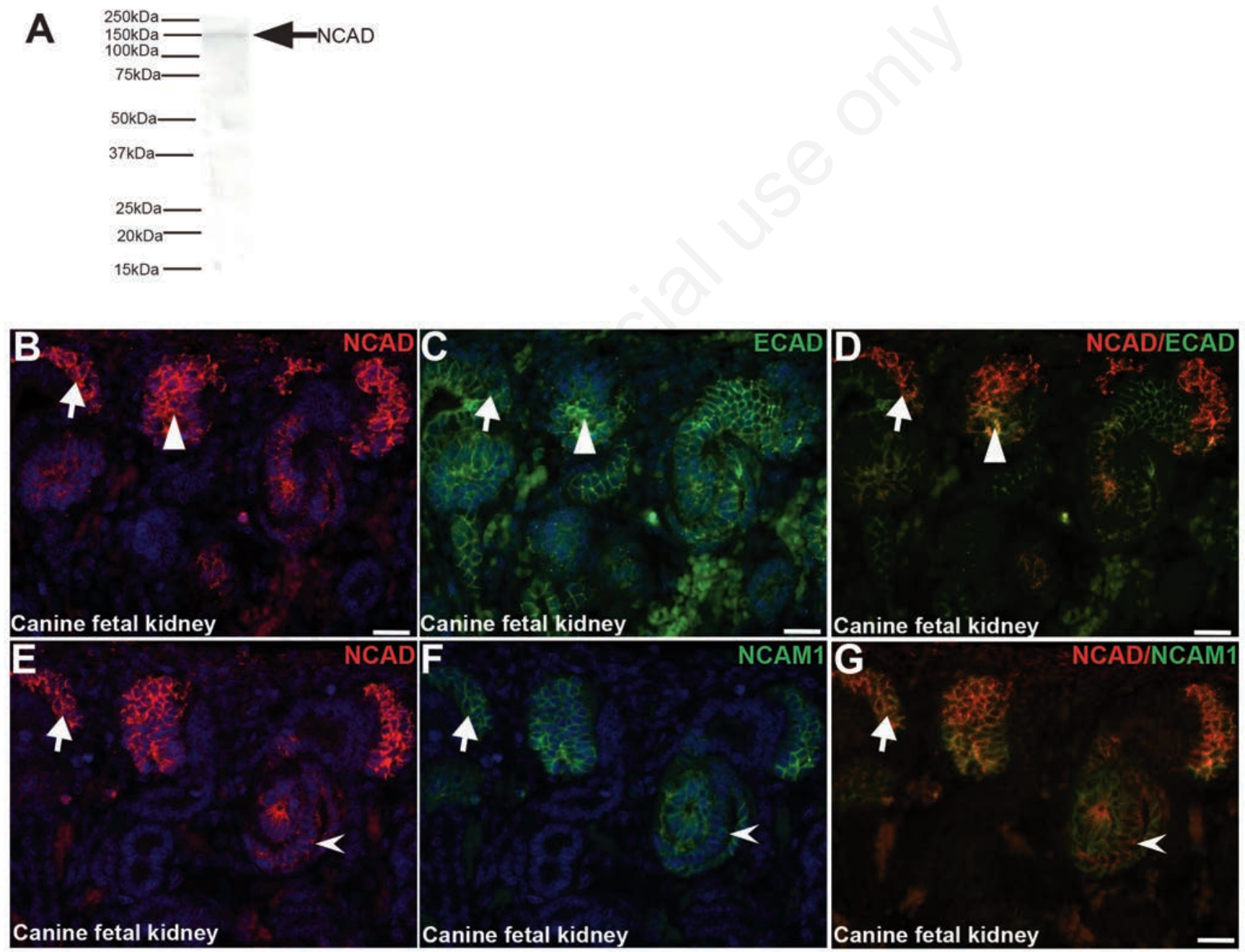

Figure 7. Expression of NCAD in the CFK. A) Whole kidney extracts were prepared from CFK and subjected to Immunoblot analysis with antiserum against NCAD antiserum. B-D) Representative co-immunofluorescence images of CFK sections with NCAD (red) and ECAD (green) antisera; nuclei were stained with DAPI; a strong NCAD signal (red) (arrow) (B) was observed on surface of NP population that did not express ECAD (green) (arrow) (C); shown also on the merged image (arrow) (D); NCAD expression (arrowhead) (B) was observed in the ECAD-positive pretubular aggregates (arrowhead) (C); also shown on the merged image (arrowhead) (D). EG) Representative co-immunofluorescence images of CFK sections with NCAD (red) and NCAM1 (green) antisera. NCAD-positive cells (red) (arrow) (E) also express strong NCAM1 signal (green) (arrow) (F); also shown on the merged image (arrow) (G); NCAD expression (concave arrowhead) (E) the S-shaped body identified by NCAM1 expression (concave arrowhead) (F); also shown on the merged image (concave arrowhead) (G). Scale bars: $20 \mu \mathrm{m}$. 
chronic kidney disease. This study reports the expression and localization of three surface markers ITGA8, NCAM1 and NCAD in the CFK. ITGA 8 was found to be expressed highly in the cell surface of canine NPs and expressed at very low levels only in a sub-population of cells in NPderived early epithelial structures Similar expression pattern of ITGA8 has been reported in mice and human fetal kidney. ${ }^{50}$ ITGA8, has been successfully used to sort and enrich NPs from the mouse and human kidney. ${ }^{46,51}$ Following the removal of the renal capsule, underlying NP population is preferentially dissociated by partial/incomplete enzymatic digestion for a short period of time. By this method, fewer cells from early epithelial derivatives are likely dissociated and leading to NP enrichment. Furthermore, to reduce contaminating interstitial cells negative selection for PDFRA has been found to be useful., ${ }^{4,51,65,66}$ Single cell RNA-sequencing profiling has revealed that NPs from human and mouse kidneys are ITGA $^{+} /$PDGFA- $^{-}$and sorting cells based on the expression of these two markers results in high enrichment of NPs..$^{51,66}$ Recently, it has been shown that the most of the ITGA $8^{+} / \mathrm{PDFGA}^{-}$population from the iPSC-derived NPs were SIX2-positive NPs that robustly generated epithelial cells of glomeruli and renal tubules following stimulation with differentiating signals. ${ }^{65}$ Future studies to determine the expression of PDGFA or other interstitial cell surface markers in CFK will be useful to select marker(s) for negative selection to minimize contaminating interstitial cells.

NCAM1 has also been utilized to enrich human NP population by magnetic-activated cell sorting and NCAM1-positive cells have been shown to have nephron-forming potential and the ability to cause renal repair following chronic renal failure. ${ }^{32,67}$ However, cells sorted based solely on NCAM1 expression contained both SIX2positive and SIX2-negative population. ${ }^{32}$ Further studies reported that $\mathrm{NCAM}^{+} / \mathrm{CD} 133^{-}$-based strategy led to better enrichment of multipotent renal stem cells as it removes $\mathrm{NCAM}^{+} / \mathrm{CD} 133^{+}$cells of early epithelial derivative structures and immature tubules. ${ }^{68}$ To remove cells from mature renal tubules negative selection for the third marker EPCAM was found to be useful. ${ }^{4,69}$ The expression of CD133 and EPCAM in the embryonic and CFK remains to be elucidated.

Canine NPs also expressed high levels of NCAD and a weak NCAD signal was also expressed in early epithelial derivates. NCAD is known to be expressed in the NP population of the fetal mouse kidney. ${ }^{52}$ Together, the results of this study suggest that high expression levels of these markers at the canine NP cell surface makes them suitable candidates for the sorting and enrichment of NP population from the developing canine kidneys.

In summary, to our knowledge, this is the first report that describes the molecular features of NPs and their derivatives in CFK and highlights the similarities and distinct features between their mouse and human counterparts. These results provide an important framework that would facilitate identification, isolation and culture of canine NPs as well as their functional characterization to develop into nephrons.

\section{References}

1. McMahon AP. Development of the Mammalian Kidney. Curr Top Dev Biol 2016;117:31-64.

2. Little MH, McMahon AP. Mammalian kidney development: principles, progress, and projections. Cold Spring Harb Perspect Biol 2012;4 pii: a008300

3. Costantini F, Kopan R. Patterning a complex organ: branching morphogenesis and nephron segmentation in kidney development. Dev Cell 2010;18:698-712.

4. Brown AC, Muthukrishnan SD, Oxburgh L. A synthetic niche for nephron progenitor cells. Dev Cell 2015;34:229-41.

5. Oxburgh L. Kidney nephron determination. Annu Rev Cell Dev Biol 2018;34:427-50.

6. Kobayashi A, Valerius MT, Mugford JW, Carroll TJ, Self M, Oliver G, et al. Six 2 defines and regulates a multipotent self-renewing nephron progenitor population throughout mammalian kidney development. Cell Stem Cell 2008;3: 169-81.

7. Brown AC, Muthukrishnan SD, Guay JA, Adams DC, Schafer DA, Fetting JL, et al. Role for compartmentalization in nephron progenitor differentiation. Proc Natl Acad Sci USA 2013;110:4640-5.

8. Boyle S, Shioda T, Perantoni AO, de Caestecker M. Cited1 and Cited2 are differentially expressed in the developing kidney but are not required for nephrogenesis. Dev Dyn 2007;236: 2321-30.

9. Georgas K, Rumballe B, Valerius MT, Chiu HS, Thiagarajan RD, Lesieur E, et al. Analysis of early nephron patterning reveals a role for distal RV proliferation in fusion to the ureteric tip via a cap mesenchyme-derived connecting segment. Dev Biol 2009;332:273-86.

10. Brunskill EW, Aronow BJ, Georgas K, Rumballe B, Valerius MT, Aronow J, et al. Atlas of gene expression in the developing kidney at microanatomic resolution. Dev Cel. 2008;15:781-91.

11. Brunskill EW, Park JS, Chung E, Chen F, Magella B, Potter SS. Single cell dissection of early kidney development: multilineage priming. Development 2014;141:3093-101.

12. Tanigawa S, Sharma N, Hall MD, Nishinakamura R, Perantoni AO. Preferential propagation of competent SIX2+ nephronic progenitors by LIF/ROCKi treatment of the metanephric mesenchyme. Stem Cell Rep 2015;5:435-47.

13. Li Z, Araoka T, Wu J, Liao HK, Li M, Lazo M, et al. 3D Culture Supports long-term expansion of mouse and human nephrogenic progenitors. Cell Stem Cell 2016;19:516-29.

14. Da Sacco S, Thornton ME, Petrosyan A, Lavarreda-Pearce M, Sedrakyan S, Grubbs BH, et al. Direct isolation and characterization of human nephron progenitors. Stem Cells Transl Med 2017; 6:419-33.

15. Takasato M, Er PX, Chiu HS, Maier B, Baillie GJ, Ferguson C, et al. Kidney organoids from human iPS cells contain multiple lineages and model human nephrogenesis. Nature 2016;536:238.

16. Kumar SV, Er PX, Lawlor KT, Motazedian A, Scurr M, Ghobrial I, et al. Kidney micro-organoids in suspension culture as a scalable source of human pluripotent stem cell-derived kidney cells. Development 2019;146. pii: dev172361.

17. Morizane R, Bonventre JV. Generation of nephron progenitor cells and kidney organoids from human pluripotent stem cells. Nat Protoc 2017;12:195-207.

18. 1Menon R, Otto EA, Kokoruda A, Zhou J, Zhang Z, Yoon E, et al. Single-cell analysis of progenitor cell dynamics and lineage specification in the human fetal kidney. Development 2018;145. pii: dev164038

19. Lindström NO, De Sena Brandine G, Ransick A, McMahon AP. Single-cell RNA sequencing of the adult mouse kidney: From molecular cataloging of cell types to disease-associated predictions. Am J Kidney Dis 2019;73:140-2.

20. Lindström NO, De Sena Brandine G, Tran T, Ransick A, Suh G, Guo J, et al. Progressive recruitment of mesenchymal progenitors reveals a time-dependent process of cell fate acquisition in mouse and human nephrogenesis. Dev Cell 2018;45:651-60. e4.

21. Lindström NO, Guo J, Kim AD, Tran T, Guo Q, De Sena Brandine G, et al. Conserved and divergent features of mesenchymal progenitor cell types within the cortical nephrogenic niche of 
the human and mouse kidney. J Am Soc Nephrol 2018;29:806-24.

22. Lindström NO, McMahon JA, Guo J, Tran T, Guo Q, Rutledge E, et al. Conserved and divergent features of human and mouse kidney organogenesis. J Am Soc Nephrol 2018;29:785-805.

23. Lindström NO, Tran T, Guo J, Rutledge E, Parvez RK, Thornton ME, et al. Conserved and divergent molecular and anatomic features of human and mouse nephron patterning. J Am Soc Nephrol 2018;29:825-40.

24. Wang P, Chen Y, Yong J, Cui Y, Wang $\mathrm{R}$, Wen L, et al. Dissecting the global dynamic molecular profiles of human fetal kidney development by single-cell RNA sequencing. Cell Rep 2018;24: 3554-67 e3.

25. Morizane R, Miyoshi T, Bonventre JV. Concise review: kidney generation with human pluripotent stem cells. Stem Cells 2017;35:2209-17.

26. Little MH, Hale LJ, Howden SE, Kumar SV. Generating Kidney from Stem Cells. Annu Rev Physiol 2019;81:335-57.

27. Bartlett PC, Van Buren JW, Neterer M, Zhou C. Disease surveillance and referral bias in the veterinary medical database. Prev Vet Med 2010;94:264-71.

28. Dillard KJ, Hytonen MK, Fischer D, Tanhuanpaa K, Lehti MS, VainioSiukola K, et al. A splice site variant in INPP5E causes diffuse cystic renal dysplasia and hepatic fibrosis in dogs. PLoS One 2018;13:e0204073.

29. Pelander L, Ljungvall I, Egenvall A, Syme H, Elliott J, Haggstrom J. Incidence of and mortality from kidney disease in over 600,000 insured Swedish dogs. Vet Rec 2015;176:656.

30. Schnaper HW. Remnant nephron physiology and the progression of chronic kidney disease. Pediatr Nephrol 2014;29:193-202.

31. Toyohara T, Mae S, Sueta S, Inoue T, Yamagishi Y, Kawamoto T, et al. Cell therapy using human induced pluripotent stem cell-derived renal progenitors ameliorates acute kidney injury in mice. Stem Cells Transl Med 2015;4:980-92.

32. Harari-Steinberg O, Metsuyanim S, Omer D, Gnatek Y, Gershon R, PriChen $\mathrm{S}$, et al. Identification of human nephron progenitors capable of generation of kidney structures and functional repair of chronic renal disease. EMBO Mol Med 2013;5:1556-68.

33. de Morais HS, DiBartola SP, Chew DJ. Juvenile renal disease in golden retrievers: 12 cases (1984-1994). J Am Vet Med Assoc 1996;209:792-7.

34. Kim J, Choi H, Lee Y, Jung J, Yeon S, Lee H. Multicystic dysplastic kidney disease in a dog. Can Vet J 2011;52:645-9.

35. Olenick CL. Congenital renal dysplasia and psychogenic polydipsia in a Bernese mountain dog. Can Vet J 1999;40:425-6.

36. Njaa BL. Kirkbride's diagnosis of abortion and neonatal loss in animals. J. Wiley \& Sons, Ltd.; 2012.

37. Pretzer SD. Canine embryonic and fetal development: a review. Theriogenology 2008;70:300-3.

38. Di Giovanni V, Walker KA, Bushnell D, Schaefer C, Sims-Lucas S, Puri P, et al. Fibroblast growth factor receptorFrs2alpha signaling is critical for nephron progenitors. Dev Biol 2015;400:82-93.

39. Puri P, Bushnell D, Schaefer CM, Bates CM. Six2creFrs2alpha knockout mice are a novel model of renal cystogenesis. Sci Rep 2016;6:36736.

40. Puri P, Phillips BT, Suzuki H, Orwig KE, Rajkovic A, Lapinski PE, et al. The transition from stem cell to progenitor spermatogonia and male fertility requires the SHP2 protein tyrosine phosphatase. Stem Cells 2014;32:74153.

41. Puri P, Schaefer CM, Bushnell D, Taglienti ME, Kreidberg JA, Yoder BK, et al. Ectopic phosphorylated creb marks dedifferentiated proximal tubules in cystic kidney disease. Am J Pathol 2018;188:84-94.

42. Puri P, Walker WH. The tyrosine phosphatase SHP2 regulates Sertoli cell junction complexes. Biol Reprod 2013;88:59.

43. Weber S, Taylor JC, Winyard P, Baker KF, Sullivan-Brown J, Schild R, et al. SIX2 and BMP4 mutations associate with anomalous kidney development. J Am Soc Nephrol 2008;19:891-903.

44. Metsuyanim S, Harari-Steinberg O, Buzhor E, Omer D, Pode-Shakked N, Ben-Hur H, et al. Expression of stem cell markers in the human fetal kidney. PLoS One 2009;4:e6709.

45. Abbate M, Brown D, Bonventre JV. Expression of NCAM recapitulates tubulogenic development in kidneys recovering from acute ischemia. Am J Physiol 1999;277:F454-63.

46. O'Brien LL, Guo Q, Lee Y, Tran T, Benazet JD, Whitney $\mathrm{PH}$, et al. Differential regulation of mouse and human nephron progenitors by the Six family of transcriptional regulators. Development 2016;143:595-608.

47. Naiman N, Fujioka K, Fujino $M$, Valerius MT, Potter SS, McMahon AP, et al. Repression of interstitial identity in nephron progenitor cells by $\operatorname{Pax} 2$ establishes the nephron-interstitium boundary during kidney development. Dev Cell 2017;41:349-65 e3.

48. Narlis M, Grote D, Gaitan Y, Boualia SK, Bouchard M. Pax2 and pax 8 regulate branching morphogenesis and nephron differentiation in the developing kidney. J Am Soc Nephrol 2007;18:1121-9.

49. Brophy PD, Ostrom L, Lang KM, Dressler GR. Regulation of ureteric bud outgrowth by Pax2-dependent activation of the glial derived neurotrophic factor gene. Development 2001;128: 4747-56.

50. Muller U, Wang D, Denda S, Meneses JJ, Pedersen RA, Reichardt LF. Integrin alpha8beta1 is critically important for epithelial-mesenchymal interactions during kidney morphogenesis. Cell 1997;88:603-13

51. Taguchi A, Kaku Y, Ohmori T, Sharmin $\mathrm{S}$, Ogawa $\mathrm{M}$, Sasaki $\mathrm{H}$, et al. Redefining the in vivo origin of metanephric nephron progenitors enables generation of complex kidney structures from pluripotent stem cells. Cell Stem Cell 2014;14:53-67.

52. Recuenco MC, Ohmori T, Tanigawa S, Taguchi A, Fujimura S, Conti MA, et al. Nonmuscle myosin II regulates the morphogenesis of metanephric mesenchyme-derived immature nephrons. J Am Soc Nephrol 2015;26:1081-91.

53. Ishimine $H$, Yamakawa $N$, Sasao $M$, Tadokoro M, Kami D, Komazaki S, et al. N-Cadherin is a prospective cell surface marker of human mesenchymal stem cells that have high ability for cardiomyocyte differentiation. Biochem Biophys Res Commun 2013;438:753-9.

54. Ruf RG, Xu PX, Silvius D, Otto EA, Beekmann F, Muerb UT, et al. SIX1 mutations cause branchio-oto-renal syndrome by disruption of EYA1-SIX1DNA complexes. Proc Natl Acad Sci USA 2004;101:8090-5.

55. Eisenbrandt DL, Phemister RD. Postnatal development of the canine kidney: quantitative and qualitative morphology. Am J Anat 1979;154:179-93.

56. Hou XM, Chen X, Wang YL. [The role of Pax2 in regulation of kidney development and kidney disease].[Article in Chinese]. Yi Chuan 2011;33:931-8.

57. Bouchard M, Souabni A, Mandler M, Neubuser A, Busslinger M. Nephric lineage specification by $\operatorname{Pax} 2$ and Pax 8 . Genes Dev. 2002;16:2958-70.

58. Torres M, Gomez-Pardo E, Dressler GR, Gruss P. Pax-2 controls multiple steps of urogenital development. Development 1995;121:4057-65.

59. Clarke JC, Patel SR, Raymond RM, Jr., Andrew S, Robinson BG, Dressler GR, et al. Regulation of c-Ret in the developing 
kidney is responsive to Pax2 gene dosage. Hum Mol Genet 2006; 15:3420-8.

60. Porteous S, Torban E, Cho NP, Cunliffe H, Chua L, McNoe L, et al. Primary renal hypoplasia in humans and mice with PAX2 mutations: evidence of increased apoptosis in fetal kidneys of Pax2(1Neu) +/- mutant mice. Hum Mol Genet 2000;9:1-11.

61. Dziarmaga A, Quinlan J, Goodyer P. Renal hypoplasia: lessons from Pax2. Pediatr Nephrol 2006;21:26-31.

62. Chung GW, Edwards AO, Schimmenti LA, Manligas GS, Zhang YH, Ritter R, 3rd. Renal-coloboma syndrome: report of a novel PAX2 gene mutation. Am J Ophthalmol 2001;132:910-4.

63. Nishimoto K, Iijima K, Shirakawa T,
Kitagawa K, Satomura K, Nakamura H, et al. PAX2 gene mutation in a family with isolated renal hypoplasia. J Am Soc Nephrol 2001;12:1769-72.

64. Xu PX, Zheng W, Huang L, Maire P, Laclef C, Silvius D. Six1 is required for the early organogenesis of mammalian kidney. Development. 2003;130:3085-94.

65. Tanigawa S, Naganuma H, Kaku Y, Era T, Sakuma T, Yamamoto T, et al. Activin is superior to BMP7 for efficient maintenance of human iPSCderived nephron progenitors. Stem Cell Rep 2019;13:322-37.

66. Kaku Y, Taguchi A, Tanigawa S, Haque F, Sakuma T, Yamamoto T, et al. PAX2 is dispensable for in vitro nephron formation from human induced pluripotent stem cells. Sci Rep 2017;7:4554.

67. Pleniceanu O, Omer D, HarariSteinberg O, Dekel B. Renal lineage cells as a source for renal regeneration. Pediatr Res 2018;83:267-74.

68. Pode-Shakked N, Pleniceanu O, Gershon R, Shukrun R, Kanter I, Bucris E, et al. Dissecting stages of human kidney development and tumorigenesis with surface markers affords simple prospective purification of nephron stem cells. Sci Rep 2016;6:23562.

69. Pode-Shakked N, Gershon R, Tam G, Omer D, Gnatek Y, Kanter I, et al. Evidence of in vitro preservation of human nephrogenesis at the single-cell level. Stem Cell Rep 2017;9:279-91. 